\title{
Mediators between adverse childhood experiences and suicidality
}

\author{
Rytilä-Manninen, Minna
}

2018-03

Rytilä-Manninen , M , Haravuori , H , Fröjd , S , Marttunen , M \& Lindberg , N 2018 , ' Mediators between adverse childhood experiences and suicidality ' , Child Abuse and Neglect , vol. 77 , pp. 99-109 . https://doi.org/10.1016/j.chiabu.2017.12.007

http://hdl.handle.net/10138/301295

https://doi.org/10.1016/j.chiabu.2017.12.007

publishedVersion

Downloaded from Helda, University of Helsinki institutional repository.

This is an electronic reprint of the original article.

This reprint may differ from the original in pagination and typographic detail.

Please cite the original version. 


\title{
Mediators between adverse childhood experiences and suicidality
}

\author{
Minna Rytilä-Manninen ${ }^{\mathrm{a}, *}$, Henna Haravuori ${ }^{\mathrm{a}, \mathrm{b}}$, Sari Fröjd ${ }^{\mathrm{d}}$, Mauri Marttunen ${ }^{\mathrm{a}, \mathrm{b}}$, \\ Nina Lindberg ${ }^{c}$
}

${ }^{a}$ Adolescent Psychiatry, Helsinki University and Helsinki University Hospital, Helsinki, Finland

${ }^{\mathrm{b}}$ National Institute for Health and Welfare, Department of Health, Mental Health Units, Helsinki, Finland

${ }^{\mathrm{c}}$ Forensic Psychiatry, Helsinki University and Helsinki University Hospital, Helsinki, Finland

d University of Tampere, School of Health Sciences, Tampere, Finland

\section{A R T I C L E I N F O}

\section{Keywords:}

Adverse childhood experiences

Adolescence

Suicidality

Multiple mediation analyses

\begin{abstract}
A B S T R A C T
We investigated whether psychiatric symptomatology, impulsivity, family and social dysfunction, and alcohol use mediate the relationship between adverse childhood experiences (ACEs) and suicidality.

The study population comprised 206 adolescent psychiatric inpatients and 203 age- and gender-matched adolescents from the community. ACEs and suicidality were assessed using the Schedule for Affective Disorders and Schizophrenia for School-Age Children - Present and Lifetime version, the Life Events Checklist, and a structured background data collection sheet. Psychiatric symptomatology was measured using the Symptom Checklist - 90. Impulsivity, social dysfunction, and family dysfunction were measured using the Offer Self-Image Questionnaire, and alcohol use was assessed with the Alcohol Use Disorders Identification Test. A simple mediation test and multiple mediation analyses were conducted.

A positive direct effect of ACEs on suicidality was observed. Also seen was a positive indirect effect of ACEs on suicidality through psychiatric symptomatology, impulsivity, and family and social dysfunctions. Alcohol misuse did not, however, mediate the relationship between ACEs and suicidality. According to the multiple mediation analyses, psychiatric symptomatology was the most significant mediator, followed by impulsivity.

Psychiatric symptoms, impulsivity, and family and social dysfunctions are factors that should be taken into consideration when assessing suicidality in adolescents.
\end{abstract}

\section{Introduction}

Suicidality increases substantially from childhood into adolescence (Kessler, Borges, \& Walters, 1999), representing a major burden on health and a leading indication for psychiatric hospitalization (Isohookana, Riala, Hakko, \& Räsänen, 2012) in young people. Looking more closely, suicidality can be regarded as an umbrella term for various forms of suicidal thoughts and behaviors. Suicidal ideation refers to thoughts about engaging in behaviors intended to end one's life. It varies from fleeting thoughts about death to more extensive ideas and plans for suicide (Vander Stoep, McCauley, Flynn, \& Stone, 2009). A suicide attempt is defined as deliberately causing harm to oneself with at least some intent to die. Finally, a completed suicide is a lethal suicide attempt. All of these behaviors can be distinguished from non-suicidal self-injury (NSSI), which refers to self-injurious behavior occurring in the absence of suicidal intent (Miller, Esposito-Smythers, Weismoore, \& Renshaw, 2013). According to a nationally representative study

\footnotetext{
* Corresponding author at: Hospital District of Helsinki and Uusimaa, Kellokoski Hospital, 04500, Kellokoski, Finland.

E-mail addresses: minna.rytila-manninen@hus.fi, minna@pixoi.com (M. Rytilä-Manninen).
} 
of US adolescents by Nock et al. (2013), the lifetime prevalence of suicide ideation, plans, and attempts was $12.1 \%$, 4.0\%, and 4.1\%, respectively. Suicide accounts for $17.6 \%$ of all deaths among individuals aged 15-29 years in high-income countries, making it a leading cause of death for people in this age group.

\subsection{Suicidality and adverse childhood experiences}

Adverse childhood experiences (ACEs) comprise childhood emotional, physical, and sexual abuse, emotional and physical neglect, and various household dysfunctions (mother treated violently, substance abuse, mental illness, criminal behavior, parental separation/divorce), which are directed to an underaged individual (Felitti et al., 1998). In this study, we define childhood adverse experiences as physical and sexual abuse, witnessing intimate partner violence, parental psychiatric problems, parental alcohol or drug abuse, parental criminal behavior, and parental separation or divorce. ACEs often co-occur (Dong, Anda, Dube, Giles, Felitti, 2003; Dong et al., 2004; Isohookana et al., 2012; Rytilä-Manninen et al., 2014) and serve as risk factors for diverse social, emotional, and medical problems later in life (Anda et al., 2006; Dube et al., 2001; Dube, Anda, Felitti, Edwards, \& Croft, 2002; Dube et al., 2005, 2006). Anda et al. (2006) reported that the risks of adverse health outcomes increase in a graded fashion as the ACE score increases; regarding psychological stress, accumulation of ACEs may be more important than any specific negative experience (Kumar and George, 2013; Rasmussen, Nielsen, Petersen, Christiansen, Bielnberg, 2013)

Suicidality can be regarded as an outcome of a complex interplay between genetic, biological, psychiatric, psychological, social, and cultural factors (Hawton, Saunders, \& O'Connor, 2012). Experts emphasize diathesis-stress explanations in theoretical formulations, specifically that predisposing biological (e.g. neurotransmitter imbalance), personality (e.g. impulsivity), and cognitive vulnerabilities (e.g. impaired social problem-solving), combined with exposure to ACEs and psychopathology, increase the risk of suicidal behaviors (Hawton et al., 2012; Serafini et al., 2015). In this context, diathesis refers to an increased and long-term vulnerability to suicidal behavior as a consequence of critical levels of early-life stress leading to inappropriate stress regulation. According to the diathesis-stress model, a suicide attempt can be realized because a person is impulsive or prone to aggressive behavior, therefore being more likely to act on his/her suicidal feelings (Pelkonen, Karlsson, \& Marttunen, 2011). In line with the diathesisstress model, researchers have reported that childhood abuse and neglect substantially increase the risk of both suicidal ideation and attempted suicide in young people (Bruffaerts et al., 2010; Dube et al., 2001; Evans, Hawton, Rodham, \& Deeks, 2005; Miller et al., 2013; Thompson et al., 2012). A systematic review by Evans et al. (2005) found a strong link between childhood physical and sexual abuse and suicidal thoughts/attempted suicides in adolescence. Similarly, Miller et al. (2013) concluded that although all forms of maltreatment are associated with suicidal ideation and suicide attempts in adolescence, childhood sexual and emotional abuse might be more important risk factors than physical abuse. Additionally, there are studies that have found a strong dose-response relationship between the number of adversities or negative life events and suicidal behavior in youths and adults (Dube et al., 2001; Felitti et al., 1998; Kumar \& George, 2013; Serafini et al., 2015).

\subsection{Factors mediating the relationship between ACEs and suicidality}

ACEs shape the cognitive and affective styles that predispose young people to suicidality (Thompson et al., 2012). From the perspective of neurobiology, ACEs are linked to a variety of changes in brain structure and function and stress-responsive neurobiological systems, which, in turn, predispose young people to mental health problems (Anda et al., 2006), emotional dysregulation, non-suicidal self-injury, and suicidal ideation and behaviors (Brodsky \& Biggs, 2012) as well as increase their risk of committing suicide (Dube et al., 2001; Miller, Esposito-Smythers, Weismoore, \& Renshaw, 2013; Perez, Jennings, Piquero, \& Baglivio, 2016; Thompson et al., 2012). Although ACEs increase the risk of suicidal behavior, not all adolescents exposed to ACEs are suicidal, suggesting that this relation must be mediated (or moderated) by some additional variables. Because mental health and behavioral problems have been associated with suicide ideation (Stewart et al., 2017) and suicide attempts (Groschwitz et al., 2015; Stewart et al., 2017; Tuisku et al., 2006) as well as with exposure to adverse experiences (Anda et al., 2006; Dube et al., 2005, 2006; RytiläManninen et al., 2014), they might act as mediators. In fact, among adults, both depression and alcohol use disorders have been reported to mediate the relation between suicidality and ACEs (Dube et al., 2001, 2002; Felitti et al. 1998). Both interpersonal difficulties (Johnson et al., 2002) and family factors influence suicidal behavior in adolescents (Laukkanen, Honkalampi, Hintikka, Hintikka, \& Lehtonen, 2005; Randall, Doku, Wilson, \& Peltzer, 2014). Interestingly, in a longitudinal study by Johnson et al. (2002), interpersonal difficulties mediated the association between maladaptive parenting and offspring's later suicidality. Moreover parentchild-relationship has documented to mediate between ACEs and suicidality (Hardt, Herke, \& Schier, 2011).

\subsection{Present study}

Although ACEs are well-documented risk factors for adolescent suicidality, more information about the variables mediating this relation is needed (Pelkonen et al., 2011).

An approach based on multiple levels of analysis (Preacher \& Hayes, 2008), incorporating information from various aspects, is needed to advance the identification of potential mediators of the relationship between ACEs and suicidality. Testing several potential mediators simultaneously minimizes the risk of attributing mediational status to a single process when other relevant processes are omitted from the analysis. Simultaneous testing also allows each individual mediator to compete for variance in a specified outcome, leading to more effective identification of the putative mediation processes responsible for the development of suicidality in adolescents exposed to various ACEs (Preacher \& Hayes, 2008). 


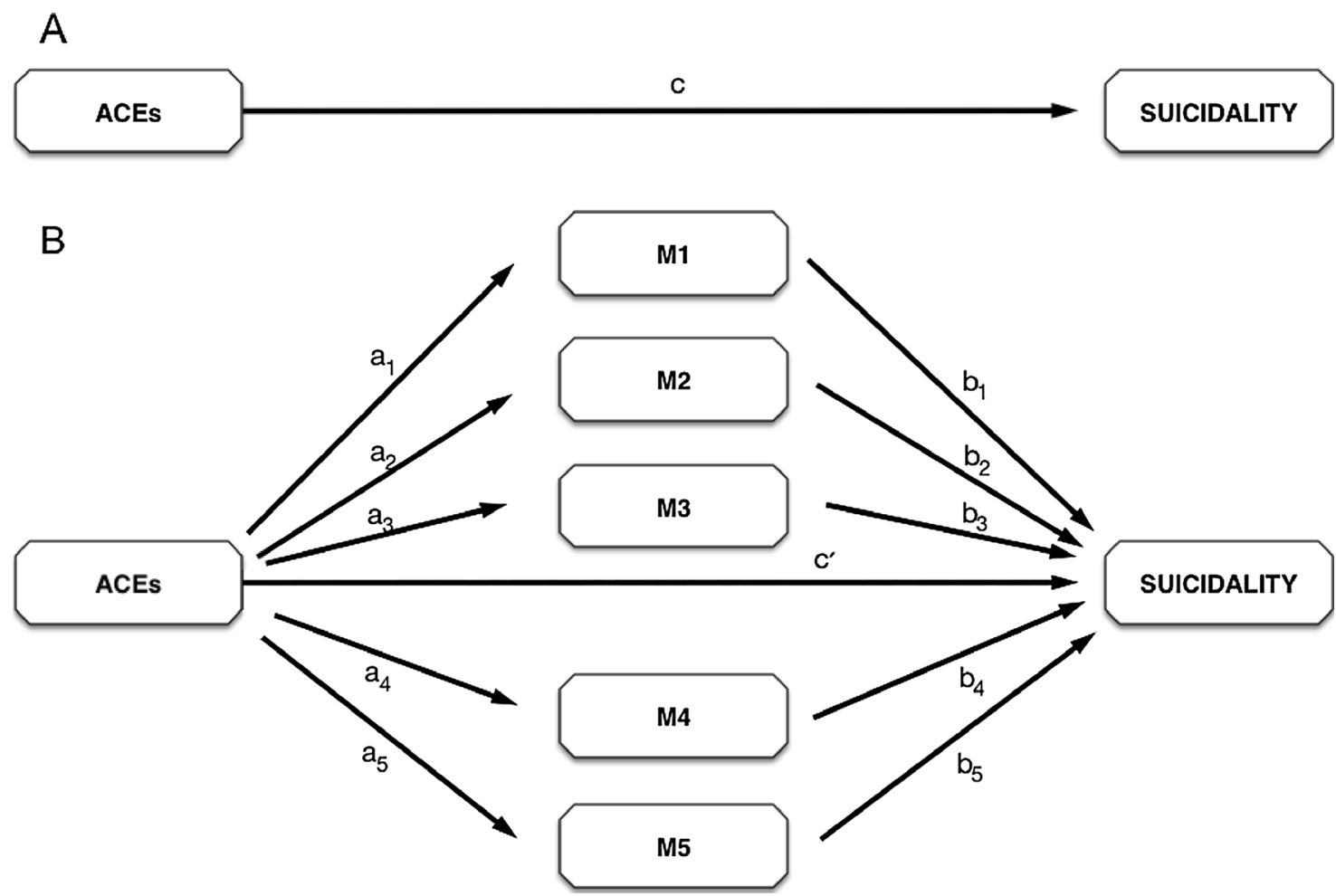

Fig. 1. Multiple mediation design with five mediators. (A) Adverse childhood experiences (ACE) affect suicidality. (B) ACE is hypothesized to exert indirect effects on suicidality through (M1) psychiatric symptoms, (M2) impulsivity, (M3) alcohol misuse, (M4) family dysfunction, and (M5) social dysfunction.

By adopting an approach using multiple levels of analysis, the present study simultaneously tested the potential role of psychiatric symptomatology, impulsivity, alcohol use, and family and social dysfunction in mediating the relationship between ACEs and suicidality among adolescents. Three primary hypotheses were tested: 1) ACEs have a direct effect on suicidality; 2) each studied mediator (psychiatric symptomatology, impulsivity, alcohol use, family dysfunction, and social dysfunction) influences the relationship between ACEs and suicidality; and 3) each potential mediator has a significant indirect effect on ACEs and suicidality when simultaneously estimated with other indirect effects (see Fig. 1).

\section{Method}

\subsection{Participants and procedure}

The Kellokoski Hospital Adolescent Inpatient Follow-Up Study (KAIFUS) is a longitudinal naturalistic study of the clinical characteristics and impact of treatment in a consecutive sample of Finnish adolescent psychiatric inpatients. This inpatient sample consists of 13- to 17-year-old adolescents referred to psychiatric hospital for the first time in their lives between 2006 and 2010 $(N=395)$. Non-eligible patients were those who had a treatment period of less than two weeks, those who showed intellectual disability, those under 13 years of age, and those with poor knowledge of the Finnish language $(\mathrm{n}=80,20.2 \%)$. Of the 315 eligible patients, $62(16.4 \%)$ declined to participate or their parents/guardians did not provide permission to participate. In 23 cases (6\%), patients or their parents discontinued the treatment period, and 24 cases (6\%) had incomplete data. Thus, the final sample comprised 206 inpatients: 60 boys and 146 girls. Non-participation was unrelated to age $(p=0.31)$, socioeconomic status ( $p=0.38)$, living situation $(p=0.58)$, substance use $(p=0.59)$, mood $(p=0.92)$, anxiety $(p=0.39)$, eating $(p=0.34)$, or conduct disorders $(p=0.09)$ as principal diagnoses, but it was associated with male gender $(p=0.02)$ and a diagnosis of psychotic disorder $(p=0.02)$. For further details, see Rytilä-Manninen et al. (2014).

The community sample comprised gender- and age-matched students from the same geographical area as the inpatient sample. They were recruited from two high schools, one vocational collage and four junior high schools. Altogether 473 students were invited to take part in the study. Of these, $202(42.5 \%)$ refused to participate and $68(14.5 \%)$ did not complete the whole study protocol despite providing consent. A total of 203 students (42.9\%, 55 boys and 148 girls)) successfully completed both the interview and selfassessments, thus comprising the final sample. No significant differences were found between completers and non-completers with regard to socioeconomic status or living situation.

The same interviews and self-questionnaires were used in the inpatient and community samples. All inpatients had a diagnosed mental disorder and many patients had comorbid conditions. Altogether 43 community adolescents (21.2\%) were diagnosed with a 
mental disorder. For details, see Rytilä-Manninen et al. (2014).

Participation was voluntary for both inpatients and community youth. All participants and their legal guardians gave written, informed consent. Permission to conduct the study was granted by the authorities of the Helsinki and Uusimaa Hospital District and the school administrations. The Ethics Committee of Helsinki University Hospital approved the study protocol.

\section{Measures}

The Schedule for Affective Disorders and Schizophrenia for School-Age Children - Present and Lifetime Version (K-SADS-PL; Kaufman et al., 1997) was used to assess psychiatric diagnoses and suicidality. K-SADS-PL is a semi-structured interview with good to excellent test-retest reliability and high concurrent validity and inter-rater agreement (Kaufman et al., 1997).

Data concerning socioeconomic status (SES) and living situation were gathered using a structured background data collection sheet. SES was assessed by the question: What is your father's occupation? If the adolescent lived with his/her biological mother, we recorded the mother's occupation. Family's SES was classified as high if the primary guardian was a self-employed worker or upperlevel employee, as middle if the guardian was a lower-level employee or manual worker, and as low if the guardian was unemployed, retired, or a student (Classification of Social Economy Status, 1989). Living situation was classified as a nuclear family if the adolescent lived with both of the biological parents, as another type of family if the adolescent lived with only one primary guardian (due to parental death or divorce) or in a blended family, and as foster care if the adolescent had been placed in a detention home.

Suicidality was measured using four questions included in the K-SADS-PL interview. The questions cover a) thoughts of death (rating: 1 = the person had not experienced thoughts of death; $2=$ the person had experienced transient thoughts of death; $3=$ the person had experienced recurrent thoughts of death); b) suicidal ideation (rating: $1=$ the person had not thought of suicide; $2=$ the person had occasionally thought of suicide; $3=$ the person had often thought of suicide as well as a specific method of carrying it out); c) suicidal acts: seriousness of the act (rating: $1=$ the person had no history of suicide attempts; $2=$ the person had made one or more suicide attempts, but was ambivalent regarding an actual wish to die; $3=$ the person had made one or more suicide attempts with a definite suicidal intent); d) suicidal acts: medical lethality of the act (rating 1 = the person had no history of suicide attempts; $2=$ the suicide attempt/s were not regarded as life-threatening, the person had showed transient and mild medical symptoms; $3=$ the suicide attempt/s had been potentially life-threatening, e.g. unconsciousness). For cross-tabulation, the rating was categorized as negative if the item was scored 1 or 2 , and positive if it was scored 3. Based on a previous study by Tuisku et al. (2006), the suicidality sum score was created by summing up the scores of these four questions. Thus, the suicidality sum score can range from 4 ( = the person showed no suicidality) to 12 ( = the person showed extreme suicidality).

Data concerning ACEs were gathered using a structured background data collection sheet, the K-SADS-PL interview screening section for PTSD, and the Life Events Checklist (LEC; Johnson and McCutcheon, 1980). Adolescents were asked whether their parents were divorced (no/yes), and whether their mother or father suffered from psychiatric or substance use problems requiring professional help (no/yes). Parents' criminality was assessed using the LEC question: "Have your parents ever been arrested or suspected or judged for a criminal offence?" (no/yes). The information about witnessing intimate partner violence (no/yes) and exposure to physical (no/ yes) or sexual abuse (no/yes) was based on the K-SADS-PL interview. Choosing the above-mentioned variables was based on earlier ACE studies (Anda et al., 2006; Dong et al., 2003; Dong et al., 2004; Dube et al., 2001, 2002, 2005, 2006). The same questions have been used in our previous study concerning adolescents' ACEs (Rytilä-Manninen et al., 2014).

The accumulation of different ACE categories was described by creating the ACE total score, which can range from zero (the person had not been exposed to any studied ACE categories) to seven (the person had been exposed to all studied ACE categories). The mean ACE total score was 2.2 (SD 1.6) for inpatients and 0.6 (SD 1.0) for adolescents in the community ( $<<0.001)$.

Psychiatric symptomatology was measured using the Symptom Checklist-90 (SCL-90; Derogatis, Lipman, \& Covi, 1973), a selfreport measure for people aged 13 years or older. It consists of 90 items, which measure subjective symptoms on nine primary symptom dimensions. Items are rated on a five-point Likert scale of distress, ranging from "not at all" (0) to "extremely" (4). Thus, the sum score can range from 0 to 360 . The reference period for the symptoms is the last two weeks. The psychometric properties of SCL90 have been shown to be good for adolescents, and it is a useful tool for assessing overall psychopathology in adolescents (RytiläManninen et al., 2016). In this study, Cronbach alphas ranged from 0.800 (paranoid ideation) to 0.943 (depressive disorders) among inpatients and from 0.749 (phobic anxiety) to 0.908 (depressive disorders) in the community sample. For further analyses, the SCL-90 sum score was used.

Impulsivity, family dysfunction, and social dysfunction were measured with the Offer Self-Image Questionnaire (OSIQ-R; Offer, Ostrov, Howard, \& Dolan, 1992), which is a 129-item personality test for adolescents aged between 13 and 18 years. The questionnaire assesses psychological adjustment based on psychodynamic growth and developmental theory. The above-mentioned three dimensions of self-image are known to be risk factors for adolescents' suicidality (Cetin, 2001; Laukkanen et al., 2005). Items are rated on a six-point Likert scale, ranging from "describes me very well" (1) to "does not describe me at all" (6). OSIQ-R comprises 12 component scales, but in this study only those described below were used. Impulse control is a nine-item scale to measure whether the adolescent can handle pressure. The scale score can range from 9 to 54. Higher scores suggest that a teenager has a low frustration tolerance and often acts on impulse. In this study, the Cronbach alpha for this scale was 0.659 in the inpatient sample and 0.629 in the community sample. Social functioning is a nine-item scale used to assess patterns of interpersonal relationships and friendships. The scale score can range from 9 to 54 . Higher scores indicate that a teenager is unable to have and maintain close relationships with same-aged individuals and feels uncomfortable when socializing with peers. In this study, the Cronbach alpha for this scale was 0.819 for inpatients and 0.729 for young people in the community. Family functioning is a 19-item scale focusing on an adolescent's feelings about and relationships with his/her parents as well as on the emotional atmosphere at home. The scale score can range from 19 to 
114. Higher scores indicate that the adolescent feels that there is tension at home, that the relationships are problematic, and that he/ she is not getting support from parents. In this study, the Cronbach's alpha for this scale was 0.842 in the inpatient sample and 0.837 in the community sample. The OSIQ has been widely used and validated for Finnish adolescents (Laukkanen, Peiponen, Halonen, Aivio, \& Viinamäki, 1999; Laukkanen, Halonen, Aivio, Viinamäki, \& Lehtonen, 2000).

Alcohol use was self-assessed with the Alcohol Use Disorders Identification Test (AUDIT; Saunders, Aasland, Babor, de la Fuente, \& Grant, 1993), which includes 10 items scored from 0 to 4. Thus, the AUDIT sum score can range from 0 to 40 . Self-assessment has shown good psychometric properties (Reinert \& Allen, 2002). In adolescents, compared against the DSM-IV diagnosis of alcohol use disorders, AUDIT has demonstrated optimal performance at a cut-off score of 4 (Chung et al., 2000). In this study, the Cronbach alpha was 0.914 for inpatients and 0.862 for the community sample.

\section{Data analysis}

To analyze the differences between young people as inpatients and in the community in suicidality sum score and in all five mediators (psychiatric symptomatology, impulsivity, alcohol use, family dysfunction, and social dysfunction), an independent samples $t$-test was performed. For further analyses, inpatient and community groups were combined to provide a broader range or more variation in the factors examined: suicidality sum score, ACEs, and the tested mediators.

The relationships between the ACE total score, the four different suicidal behaviors, the suicidality sum score, and all five mediators were explored using correlation analysis. A simple mediation test was then conducted for each variable to determine direct and indirect effects of the ACE total score on suicidality. Finally, a multiple mediation analysis was conducted to test for simultaneous indirect effects of ACEs on suicidality through multiple mediators. For this, we used Preacher and Hayes' (2008) bootstrapping procedure, which is a nonparametric sampling procedure recommended for multiple mediators. The SPSS macro for multiple mediation (Preacher \& Hayes, 2008) was used for statistical analyses. It estimates the path coefficients in a multiple mediator model and generates bootstrap confidence intervals (including percentile, bias-corrected, and bias-corrected and accelerated) for total and specific indirect effects of ACEs on suicidality through one or more mediator variables. Multiple mediator modeling enables the testing of competing hypotheses within a single model, and reduces parameter bias due to omitted variables, that is, other possible mediators. Testing for significant mediation requires that bias-corrected $95 \%$ confidence intervals not overlap with zero. Age and gender were used as covariates.

\section{Results}

\subsection{Characteristics of adolescents}

Sociodemographic characteristics of inpatients and non-referred adolescents are presented in Table 1. SES of community youth was significantly higher than that of inpatients, and non-referred adolescents lived significantly more often than inpatients in nuclear families $(p<0.001)$. Inpatients, on the other hand, lived significantly more often in other types of families or in foster care $(\mathrm{p}<0.001)$.

Altogether 98 inpatients (47.6\%) and two community adolescents (1.0\%) had experienced recurrent thoughts of death. Ninetyfour inpatients $(45.6 \%)$ and one non-referred adolescent $(0.5 \%)$ had recurrent suicidal ideation. A suicide attempt was reported by 45 inpatients (21.8\%) but none of the community sample, and potentially life-threatening suicidal acts were reported by 31 inpatients $(15.0 \%)$ but none in the community sample. The mean suicidality sum score was 7.4 (SD 2.7) for inpatients and 4.2 (SD 0.5 ) for community youth ( $\mathrm{p}<0.001)$.

Psychiatric symptomatology was significantly higher among inpatients $(M=115.5, S D=71.1)$ than among adolescents in the community $(M=44.4, S D=37.2, p<0.001)$. Inpatients were also more impulsive (inpatients: $M=31.4, S D=7.3$; community: $M=24.9, S D=6.1, p<0.001$ ) and experienced more family dysfunction (inpatients: $M=56.1, S D=16.3$; community: $M=43.3$,

Table 1

Sociodemographic characteristics of inpatients and community youth.

\begin{tabular}{|c|c|c|c|}
\hline & Patients $(n=206)$ & Controls $(n=203)$ & $p$ \\
\hline Sex, n (\%) & & & 0.661 \\
\hline Girl & $146(70.9)$ & $148(72.9)$ & \\
\hline Boy & $60(29.1)$ & $55(27.1)$ & \\
\hline Age, mean (SD) & $15.05(1.24)$ & $14.88(1.23)$ & 0.160 \\
\hline Socioeconomic status, n (\%) & & & $<0.001$ \\
\hline High & $19(9.2)$ & $30(14.8)$ & \\
\hline Middle & $78(37.9)$ & 109 (53.7) & \\
\hline Low & $109(52.9)$ & $64(31.5)$ & \\
\hline Living situation, n (\%) & & & $<0.001$ \\
\hline Nuclear family & 85 (41.7) & $127(62.6)$ & \\
\hline Other type of family & $92(45.1)$ & $75(36.9)$ & \\
\hline Foster care & $27(13.2)$ & $1(0.5)$ & \\
\hline
\end{tabular}

Chi-square test was used to compare the groups. 
Table 2

Pearson correlations between both adverse childhood experiences (ACEs) (=ACE total score) and suicidality (= suicidality sum score and its subscores related to recurrent thoughts of death, suicidal ideation, and suicidal acts) and impulsivity (=OSIQ Impulse control factor total score), alcohol misuse (=AUDIT total score), psychiatric symptoms (=SCL-90 total score), social dysfunction (=OSIQ-R Social functioning factor total score), and family dysfunction (= OSIQ-R Family functioning factor total score).

\begin{tabular}{|c|c|c|c|c|c|c|}
\hline & Impulsivity & Alcohol misuse & Psychiatric symptoms & Social dysfunction & Family dysfunction & ACEs \\
\hline ACEs & $.326^{* * * * x}$ & 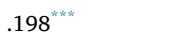 & $.404^{k \ldots k \pi}$ & $.253^{* * * *}$ & $.436^{k * * x}$ & \\
\hline Suicidality & $.509^{* * * *}$ & $.173^{* * k}$ & $.579^{k * * *}$ & $.487^{* * * *}$ & $.465^{k * k x}$ & $.402^{* * * * *}$ \\
\hline Recurrent thoughts of death & $.443^{* * * k}$ & $.044^{\mathrm{NS}}$ & $.536^{* k * *}$ & $.448^{* * * *}$ & $.413^{k+k x+2}$ & $.357^{* \ldots * * *}$ \\
\hline Suicidal ideation & $.408^{* \ldots *}$ & $.108^{*}$ & $.508^{* \ldots * *}$ & $.468^{*}$ & $.370^{* * * *}$ & $.331^{\text {***kt }}$ \\
\hline Suicidal acts: seriousness of the act & $.313^{* \ldots+k}$ & $.184^{k \text { k*k }}$ & $.303^{k \text { k*k }}$ & $.240^{\text {*iknk }}$ & $.256^{\text {kn*kx }}$ & $.194^{k * * * k}$ \\
\hline Suicidal acts: lethality of the act & $.256^{\text {think }}$ & $.180^{\text {kikik }}$ & $.169^{* k \hbar k}$ & $.119^{*}$ & $.176^{\text {k*k }}$ & $.190^{\text {kik* }}$ \\
\hline
\end{tabular}

AUDIT $=$ Alcohol Use Disorders Identification Test; SCL-90 = Symptom Checklist -90 ; OSIQ-R = Offer Self-Image Questionnaire, revised; NS = not significant.

$* \mathrm{p}<0.05$.

$* * \mathrm{p}<0.01$.

$* * * \mathrm{p}<0.001$.

$S D=13.8, p<0.001$ ) and social dysfunction (inpatients: $M=27.9, S D=8.3$; community: $M=19.9, S D=5.6, p<0.001$ ). With regard to alcohol use, no significant difference was observed between the groups (inpatients: $M=4.4, S D=6.6$; community: $M=3.5, S D=4.9, p=0.327$ ).

\subsection{Correlations}

Correlations between different variables are presented in Table 2. Significant correlations existed between suicidality variables and the ACE total score as well as psychiatric symptoms, impulsivity, alcohol use, family dysfunction, and social dysfunction; not significant was the correlation between recurrent thoughts of death and alcohol use. The strongest correlations were found between psychiatric symptoms and the suicidality sum score and between impulsivity and the suicidality sum score.

\subsection{Direct and indirect effects of cumulative ACEs on suicidality}

A positive direct effect of the ACE total score on suicidality was observed (Table 3). Additionally, a positive indirect effect of the ACE sum score on suicidality through psychiatric symptoms, impulsivity, family dysfunction, and social dysfunction was seen. All of these variables had partial mediation effects. Alcohol use, by contrast, did not affect the relationship between the ACE total score and suicidality and was excluded from further analyses. Age and gender had no significant impact on results.

\subsection{Multiple mediation model of the effect of cumulative ACEs on suicidality}

In the multiple mediation model $\left(\mathrm{R}^{2}=0.434\right.$, adjusted $\left.\mathrm{R}^{2}=0.462\right)$, the total effect of the ACE total score on suicidality was $0.754(S E=0.080 ; t=9.548 ; p<0.001)$, whereas the direct effect was 0.369 (SE $=0.077 ; t=4.770 ; p<0.001)$. The difference between the total effect and the direct effect is the total indirect effect of the ACE total score on suicidality through four mediators; its point estimate was 0.385 (Table 4). The bias-corrected bootstrap 95\% confidence interval was $0.282-0.498$, reflecting that psychiatric symptoms, impulsivity, and family and social dysfunctions together mediated the effects of ACEs on suicidality.

However, the specific indirect effects indicated that only psychiatric symptoms and impulsivity were significant mediators (Table 4). To determine whether these indirect effects differed significantly from each other, the pairwise contrast was examined. Pairwise contrast of the indirect effects showed that the specific indirect effect through psychiatric symptoms was larger than it was through impulsivity. Further, the specific indirect effect through psychiatric symptoms was larger than it was through family dysfunction or social dysfunction. However, impulsivity did not differ from social dysfunction or family dysfunction in terms of magnitude, despite impulsivity being significantly different from zero, while the other variables were not. Neither did social dysfunction differ from family dysfunction. Including age and gender as covariates in the analyses had no significant effects on results.

\section{Discussion}

We examined the impact of cumulative ACEs on suicidality as well as the possible mediating effects of psychiatric symptoms, impulsivity, alcohol use, and family and social dysfunctions on the relationship between ACEs and suicidality in adolescents.

As expected, the cumulative ACE score had a direct effect on suicidality. This finding is in line with previous research on suicidal ideation and suicide attempts (Bruffaerts et al., 2010; Dube et al., 2001; Evans et al., 2005; Isohookana et al., 2012; Miller, EspositoSmythers, Weismoore, \& Renshaw, 2013; Thompson et al., 2012). Psychiatric symptoms, impulsivity, family dysfunction, and social dysfunction each mediated the association between ACEs and suicidality. In our sample, alcohol use was not a significant mediator, although substance abuse has been related to suicidal ideation, suicide attempts, and completed suicides in adolescents in many earlier studies (e.g. Groleger, Tomori, \& Kocmur, 2003) and also to ACEs (Rothman, Edwards, Heeren, \& Hingson 2008). There are also opposite findings (Bolton, Belik, Enns, Cox, Sareen, 2008; Cluver, Orkin, Boyes, \& Sherr 2015; Hardt et al., 2011). For example 
Table 3

Simple mediation tests of the effects of adverse childhood experiences (ACEs) on suicidality through five mediators: impulsivity, alcohol misuse, psychiatric symptoms, social dysfunction, and family dysfunction.

\begin{tabular}{|c|c|c|c|c|c|c|c|c|c|c|}
\hline \multirow[t]{2}{*}{ Impulsivity } & \multirow{2}{*}{$\begin{array}{l}\text { R-sq } \\
\text { Adj R-sq }\end{array}$} & \multicolumn{3}{|l|}{0.3341} & \multicolumn{3}{|c|}{ Product of coefficients $=$ Sobel } & \multirow[t]{2}{*}{$95 \% \mathrm{CI}$} & \multirow[t]{2}{*}{ BC 95\% CI } & \multirow[t]{2}{*}{ BCa $95 \%$ CI } \\
\hline & & 0.3307 & & & & & & & & \\
\hline Path* & Coefficient & SE & $\mathrm{t}$ & $\mathrm{p}$ & SE & $\mathrm{Z}$ & $\mathrm{p}$ & & & \\
\hline a & 1.5805 & 0.2337 & 6.7638 & 0 & & & & & & \\
\hline $\mathrm{b}$ & 0.1454 & 0.0155 & 9.3868 & 0 & & & & & & \\
\hline $\mathrm{c}$ & 0.721 & 0.0785 & 9.1863 & 0 & & & & & & \\
\hline$c^{\prime}$ & 0.4912 & 0.075 & 6.553 & 0 & & & & & & \\
\hline$a b$ & 0.2298 & & & & 0.0418 & 5.4972 & 0 & $.1539-.3135$ & $.1574-.3189$ & $.1566-.3175$ \\
\hline \multirow[t]{2}{*}{ Alcohol misuse } & R-sq & 0.1675 & & & & & & & & \\
\hline & Adj R-sq & 0.1632 & & & & & & & & \\
\hline $\mathrm{a}$ & 0.743 & 0.1877 & 3.9589 & 0.0001 & & & & & & \\
\hline $\mathrm{b}$ & 0.0433 & 0.0208 & 2.0867 & 0.0376 & & & & & & \\
\hline $\mathrm{c}$ & 0.6613 & 0.0772 & 8.561 & 0 & & & & & & \\
\hline$c^{\prime}$ & 0.6291 & 0.0784 & 8.0198 & 0 & & & & & & \\
\hline$a b$ & 0.0322 & & & & 0.0174 & 1.8502 & 0.0643 & $-.0020-.0826$ & $.0004-.0886$ & $.0011-.0914$ \\
\hline \multirow[t]{2}{*}{ Psychiatric symptoms } & R-sq & 0.3742 & & & & & & & & \\
\hline & Adj R-sq & 0.371 & & & & & & & & \\
\hline $\mathrm{a}$ & 17.2872 & 1.9807 & 8.7277 & 0 & & & & & & \\
\hline $\mathrm{b}$ & 0.0187 & 0.0017 & 11.2229 & 0 & & & & & & \\
\hline c & 0.6731 & 0.0749 & 8.9865 & 0 & & & & & & \\
\hline$c^{\prime}$ & 0.3498 & 0.0713 & 4.9083 & 0 & & & & & & \\
\hline $\mathrm{ab}$ & 0.3233 & & & & 0.0468 & 6.9018 & 0 & $.2439-.4126$ & $.2465-.4160$ & $.2445-.4133$ \\
\hline \multirow[t]{2}{*}{ Social dysfunction } & R-sq & 0.3352 & & & & & & & & \\
\hline & Adj R-sq & 0.3317 & & & & & & & & \\
\hline $\mathrm{a}$ & 1.3812 & 0.2576 & 5.3621 & 0 & & & & & & \\
\hline $\mathrm{b}$ & 0.1346 & 0.014 & 9.5811 & 0 & & & & & & \\
\hline c & 0.711 & 0.0788 & 9.0284 & 0 & & & & & & \\
\hline$c^{\prime}$ & 0.5251 & 0.0734 & 7.153 & 0 & & & & & & \\
\hline$a b$ & 0.1859 & & & & 0.0397 & 4.6867 & 0 & $.1137-.2719$ & $.1170-.2773$ & $.1143-.2735$ \\
\hline \multirow[t]{2}{*}{ Family dysfunction } & R-sq & 0.2764 & & & & & & & & \\
\hline & Adj R-sq & 0.2725 & & & & & & & & \\
\hline $\mathrm{a}$ & 4.5066 & 0.4994 & 9.0237 & 0 & & & & & & \\
\hline $\mathrm{b}$ & 0.0521 & 0.0079 & 6.6089 & 0 & & & & & & \\
\hline c & 0.7525 & 0.0802 & 9.3768 & 0 & & & & & & \\
\hline$c^{\prime}$ & 0.5175 & 0.0839 & 6.1665 & 0 & & & & & & \\
\hline$a b$ & 0.235 & & & & 0.044 & 5.3437 & 0 & $.1481-.3343$ & $.1492-.3371$ & $.1480-.3342$ \\
\hline
\end{tabular}

$\mathrm{BC}=$ bias-corrected; $\mathrm{BCa}=$ bias-corrected and accelerated; 5000 bootstrap samples.

"Paths: $\mathrm{a}=$ effect of the ACE total score on the mediator; $\mathrm{b}=$ effect of the mediating variable on suicidality sum score; $\mathrm{c}=$ overall effect of the ACE total score on suicidality sum score; $c^{\prime}=$ effect of the ACE total score on suicidality sum score controlling for mediator; ab $=$ the amount of mediation $=$ indirect effect.

Cluver et al. (2015) have reported that mental health, but not drug or alcohol misuse, mediates the relationships between the cumulative ACE score and suicidality. Accordingly, Hardt et al. (2011) found that alcohol abuse had no relationship between ACEs and suicidality. They explained this finding that alcohol use is perhaps more an indicator rather than a causal link in the development of suicidality. The most probable explanation for our finding is that, in Finland, adolescents with severe alcohol misuse are typically referred to specialized units for substance users, and thus, our sample lacked individuals with substantial alcohol problems.

Our findings that both family dysfunction and social dysfunction are mediators are congruent with Johnson et al. (2002), who found that interpersonal difficulties during adolescence mediated the association between ACEs and suicide attempts in early adulthood. Our finding is also congruent with the study by Hardt et al. (2011), which reported that missing parental warmth or love, is a powerful predictor for suicidal thoughts of offspring experienced ACEs, and in turn, one or both parents showing warmth serves as a protective factor against suicidal thoughts.

Our result of family dysfunction serving as a mediator also supports the theory that while some stress in childhood or adolescence is normal and even necessary for the development of healthy coping mechanisms and problem-solving skills, ACEs may become "toxic" when there is strong, frequent, or prolonged activation of the body's stress response system in the absence of the buffering protection of a supportive parental (at least one parent) relationship (Shonkoff and Garner, 2012; Center on the Developing Child at Harvard University, 2016). Our finding that social dysfunction mediated the relationship of ACEs and suicidality also supports earlier findings from neurobiology about numerous effects of childhood stress on brain and physical systems and represents a common pathway for a variety of important long-term behavioral and social problems (Anda et al., 2006). Given this result, one could assume that ACEs may disrupt social relations and hamper the development of social skills, thus hindering the adolescents ability to maintain normal, healthy social relationships with peers. Without these skills and abilities, the adolescent may become socially isolated, contributing to the onset of suicidal behavior. From the developmental viewpoint, adolescence is a period in which dependence on parents shifts towards dependence on peers (Collins \& Laursen, 2004). Parents nonetheless remain a vital source of social and emotional support. In fact, constant and serious family conflicts can often be seen as a continuation of poor relations rather than a 
Table 4

Multiple mediation test of the effect of adverse childhood experiences (ACEs) on suicidality through psychiatric symptoms, impulsivity, social dysfunction, and family dysfunction.

\begin{tabular}{|c|c|c|c|c|c|c|c|c|c|}
\hline \multirow[t]{3}{*}{ Tests } & \multirow{3}{*}{$\begin{array}{l}\text { Point } \\
\text { estimate }\end{array}$} & \multirow{3}{*}{$\begin{array}{l}\text { Product of } \\
\text { coefficient } \\
S E\end{array}$} & \multicolumn{7}{|c|}{ Bootstrapping } \\
\hline & & & \multirow[b]{2}{*}{$\mathrm{Z}$} & \multicolumn{2}{|c|}{ Percentile 95\% CI } & \multicolumn{2}{|c|}{ BC 95\% CI } & \multicolumn{2}{|c|}{ BCa $95 \%$ CI } \\
\hline & & & & Lower & Upper & Lower & Upper & Lower & Upper \\
\hline & & & \multicolumn{7}{|c|}{ Indirect effects } \\
\hline Psychiatric symptoms & 0.2310 & 0.0498 & $4.6394^{3 * * * t}$ & .1310 & .3375 & .1340 & .3426 & .1319 & .3386 \\
\hline Impulsivity & 0.0819 & 0.0329 & $2.4904^{*}$ & .0109 & .1663 & .0131 & .1710 & .0156 & .1749 \\
\hline Social dysfunction & 0.0369 & 0.0273 & $1.3514^{\mathrm{NS}}$ & -0.0239 & 0.1109 & -0.0232 & 0.1112 & -0.0232 & 0.1112 \\
\hline Family dysfunction & 0.0353 & 0.0383 & $0.9219^{\mathrm{NS}}$ & -0.0507 & 0.1310 & -0.0466 & 0.1357 & -0.0457 & 0.1367 \\
\hline \multirow[t]{2}{*}{ Total } & 0.3851 & 0.0572 & $6.7348^{* * * *}$ & .2880 & .4979 & .2879 & .4979 & .2851 & .4949 \\
\hline & & & \multicolumn{7}{|l|}{ Contrast } \\
\hline Psychiatric symptoms vs. Impulsivity & 0.1491 & 0.0630 & 2.3647 & .0086 & .2854 & .0070 & .2844 & .0027 & .2797 \\
\hline Psychiatric symptoms vs. Social dysfunction & 0.1941 & 0.0629 & 3.0882 & .0482 & .3378 & .0533 & .3435 & .0488 & .3393 \\
\hline Psychiatric symptoms vs. Family dysfunction & 0.1957 & 0.0637 & 3.0738 & .0595 & .3362 & .0623 & .3406 & .0609 & .3394 \\
\hline Impulsivity vs. Social dysfunction & 0.0450 & 0.0458 & 0.9834 & -0.0688 & 0.1605 & -0.0624 & 0.1651 & -0.0602 & 0.1693 \\
\hline Impulsivity vs. Family dysfunction & 0.0466 & 0.0553 & 0.8437 & -0.0906 & 0.1832 & -0.0901 & 0.1851 & -0.0884 & 0.1867 \\
\hline Social dysfunction vs. Family dysfunction & 0.0016 & 0.0522 & 0.0309 & -0.1246 & 0.1294 & -0.1330 & 0.1236 & -0.1329 & 0.1245 \\
\hline
\end{tabular}

$\mathrm{BC}=$ bias corrected; $\mathrm{BCa}=$ bias corrected and accelerated; 5000 bootstrap samples.

NS $=$ not significant.

$* \mathrm{p}<0.05$.

$* * * \mathrm{p}<0.001$.

special characteristic of adolescence itself (Collins \& Laursen, 2004). The mediating role of family dysfunction highlights the importance of including the family in the treatment of suicidal adolescents. It is important to strengthen the relationship between parent (s) and offspring, which, in turn, helps the adolescent to increase his/her ability to plan for the future, regulate behavior, and develops skills to adapt to changing circumstances. This foundation is known as resilience (Center on the Developing Child at Harvard University, 2016).

The multiple mediation analyses where the mediator variables competed against each other revealed that perceived psychiatric symptoms were the most significant mediator between ACEs and suicidality. Youth suicide has been found to be associated with many psychiatric disorders, especially depression. Major depression has been estimated to be present in at least 35\% of adolescent suicides (Spirito \& Esposito-Smythers, 2006). Many studies examining the relationship between ACEs and suicidality have used mental health problems, such as depression, as controlling variables, showing a trend of a mediating effect of mental health problems on the relationship between ACEs and suicidality (Johnson et al., 2002; Thompson et al., 2012). These studies provide some evidence that psychiatric symptoms or depressive disorders partially mediate the link between ACEs and suicidality.

In this study, the other significant mediator was impulsivity. This finding receives some support from the work of Zouk, Tousingnant, Sequin, Lesage, and Turecki (2006), who found in their retrospective study that impulsive suicide completers were more likely to have a history of childhood abuse. Stressful life events and early adversity have been observed to increase the risk of suicidality via their influence on child brain structures, stress regulation systems, and serotonin levels (Braquehais, Oquendo, BacaGarcía, \& Sher, 2010). Furthermore, low serotonin function has been associated with impulsive and aggressive behaviors in adolescents with different psychiatric diagnoses including depression (Braquehais et al., 2010). Impulsivity has been assumed to be related to trauma in two different ways. First, as a consequence of childhood trauma (mainly as a personality trait), it could be an acquired inability of the brain to inhibit some negative actions. Second, impulsivity (as a trait or state) could also be seen as a risk factor for exposure to traumas and the development of post-traumatic stress disorder (Braquehais et al., 2010).

Based on this research, we can presume that ACEs have an independent effect on suicidality. Moreover, ACEs increase the risk of suffering from psychiatric symptoms in adolescence, and these symptoms heighten the risk of suicidality. Further, ACEs may increase impulsivity, thus increasing the risk of suicidality by mediating between ACEs and suicidality. Family and social dysfunctions have mediating effects on the relationship between ACEs and suicidality, but psychiatric symptoms and impulsivity attenuated these effects in the multiple mediating test.

Our study supports previous research on the developmental pathway from childhood adversities through psychological problems to suicidality. Brain development is active in the early years, and although genes provide the brain's basic blueprint, experiences modify the neurobiological development of a child. To learn to cope with stress is a vital part of childhood development. When a child's stress response system is activated in a supportive environment, the physiological effects are buffered and returned to baseline, which, in turn, results in the development of a healthy response system. If the stress is strong and frequent without protective parental support, the prolonged activation of the stress response systems disrupts neurodevelopment. As a result, the child's ability to cope with negative or disruptive emotions is impaired, leading to emotional dysregulation and impulsivity. During adolescence the young can adopt negative coping mechanisms such as suicidal behavior (Hawton et al., 2012; Center on the Developing Child at Harvard 
University, 2014; Shonkoff \& Garner, 2012). Therefore, it is important to recognize and prevent stressful adverse experiences as early as possible. In cases of severe problems, rehabilitation and attempts to reduce the long-term suffering and disabilities associated with adversities are needed (Braquehais et al., 2010).

According to the systematic review by Ougrin, Tranah, Stahl, Moran, and Asarnow (2015), therapies with the greatest impact on treating adolescent suicidal behavior are dialectical behavior therapy for adolescents (DBT-A), cognitive-behavioral therapy (CBT), and mentalization-based therapy (MBT). DBT-A improves adolescents' global and social adjustment and personality functioning. In DBT-A, also parents or other caregivers participate in the skills training groups (Mehlum et al., 2016). CBT, particularly its suicide prevention model (CBT-SP), focuses on developing cognitive, behavioral, and interactional skills that will enable the adolescent to refrain from further suicidal behavior. Parents participate in family sessions, which focus specifically on suicide risk reduction strategies (Stanley et al., 2009). MBT, which is based on attachment theory and psychodynamic principles, has a high degree of structure and a clear treatment goal of improving patients' metalizing skills. The parent-child relationship is also regarded as an important treatment target (Beck et al., 2016). Parent-child interaction therapy (PCIT) and child-parent psychotherapy (CPP) are evidence-based treatment modalities to prevent and manage ACEs and their impact on intra-familial relationships (Center on the Developing Child at Harvard University, 2016).

\subsection{Study strengths and limitations}

The main strength of this study was that we were able to collect a consecutive sample of adolescent inpatients. It is also noteworthy that our study included a gender- and age-matched community sample of students from the same geographical area as the inpatient group. The protocols were identical to both samples, improving the study's validity (Du Fort, Newman, \& Bland, 1993). We used reliable and valid semi-structured K-SADS-PL interviews to determine DSM-IV-based psychiatric diagnoses and suicidality. A structured background data collection sheet allowed us to systematically collect the same background information from both samples.

The study also has some obvious limitations. Recall bias may have occurred because some data collection relied on retrospective reports. The Adverse Childhood Experience (ACE) Questionnaire was not used, as it is designed for adults and our study comprises underaged individuals. We also used categorical ACE variables, and thus, the influence of a single ACE was not taken into account. The gender distribution was suboptimal; the samples were female-dominated. Also attrition in inpatients was related to male gender and a psychotic disorder diagnosis. Data on psychiatric symptoms were based on the SCL-90 self-report questionnaire, which has the strongest association with depressive symptoms (Rytilä-Manninen et al., 2016). Therefore, externalizing symptoms may have been underestimated. Additionally, in the index hospital district, patients who suffer from severe substance use problems or severe eating disorders are referred to specialized units, not to the psychiatric hospital. In Finland, most adolescents with serious conduct disorders are referred to child welfare services, not to mental health services.

\section{Conclusion}

ACEs have an independent effect on the suicidality of adolescents. Of the studied mediators, psychiatric symptoms and impulsivity produce the most powerful mediation effects. Family dysfunction and social dysfunction have lesser, albeit significant effects on suicidality as single mediators. Thus, interpersonal problems should also be taken into account when assessing suicidality of an adolescent.

This study was funded by the Finnish Government through special research grants to the hospital district, awarded to Minna Rytilä-Manninen.

\section{References}

Anda, R., Felitti, V., Bremner, J. D., Walker, J., Whitfield, C., Perry, B., et al. (2006). The enduring effects of abuse and related adverse experiences in childhood: A convergence of evidence from neurobiology and epidemiology. European Archives of Psychiatry and Clinical Neuroscience, 256, $174-186$.

Beck, E., Bo, S., Gondan, M., Poulsen, S., Pedersen, L., Pedersen, J., et al. (2016). Mentalization-based treatment in groups for adolescents with borderline personality disorder (BPD) or subtreshold BPD versus treatment as usual (M-GAB): Study protocol for a randomized controlled trial. Trials, 17, 314. http://dx.doi.org/10. 1186/s13063-016-1431-0 [Retrieved from].

Bolton, J., Belik, S., Enns, M., Cox, B., \& Sareen, J. (2008). Exploring the correlates of suicide attempts among individuals with major depressive disorder; findings from the national epidemiologic survey on alcohol and related conditions. Journal of Clinical Psychiatry, 69, 1139-1149.

Braquehais, D., Oquendo, M., Baca-Garcia, E., \& Sher, L. (2010). Is impulsivity a link between childhood abuse and suicide? Comprehensive Psychiatry, 51, 121-129.

Brodsky, B., \& Biggs, E. (2012). Adverse childhood experiences and suicidal behavior. Suicidology, 3, 16-21.

Bruffaerts, R., Demyttenaere, K., Borges, G., Haro, J., Chiu, W., Hwang, I., et al. (2010). Childhood adversities as risk factors for onset and persistence of suicidal behavior. British Journal of Psychiatry, 197, 20-27.

Center on the Developing Child at Harvard University (2014). Key concepts: Toxic stress. Retrieved from http://developingchild.harvard.edu.

Center on the Developing Child at Harvard University (2016). Applying the Science of Child Development in Child Welfare Systems. Retrieved from http://www. developinchild.harvard.edu.

Cetin, F. (2001). Suicide attempts and self-image among Turkish adolescents. Journal of Youth and Adolescence, 30, 641-650.

Chung, T., Colby, S., Barnett, N., Rohsenow, D., Spirito, A., \& Monti, P. (2000). Screening adolescents for problem drinking: Performance of brief screening against DSM-IV alcohol diagnoses. Journal of Studies on Alcohol, 61, 579-587.

Classification of Social Economy Status (1989). National authority for collecting and compiling statistics on various fields of society and economy. Handbooks. in Finnishz.

Cluver, L., Orkin, M., Boyes, M., \& Sherr, L. (2015). Child and adolescent suicide attempts, suicidal behavior, and adverse childhood experiences in South Africa: A prospective study. Journal of Adolescent Health, 57, 52-59.

Collins, W., \& Laursen, B. (2004). Parent-adolescent relationships and influences. In S. Lerner, \& L. Steinberg (Eds.). Handbook of adolescent psychology. New Jersey: John Wiley \& Sons Hoboken. 
Derogatis, L., Lipman, R., \& Covi, L. (1973). SCL-90: An outpatient psychiatric rating scale- preliminary report. Psychopharmacology, 9, 13-28.

Dong, M., Anda, R., Dube, S., Giles, W., \& Felitti, V. (2003). The relationship of exposure to childhood sexual abuse to other forms of abuse, neglect, and household dysfunction during childhood. Child Abuse \& Neglect, 27, 625-639.

Dong, M., Anda, R., Felitti, V., Dube, S., Williamson, D., Thompson, T., et al. (2004). The interrelatedness of multiple forms of childhood abuse, neglect, and household dysfunction. Child Abuse \& Neglect, 28, 771-784.

Du Fort, G., Newman, S., \& Bland, R. (1993). Psychiatric comorbidity and treatment seeking: Sources of selection bias in the study of clinical populations. Journal of Nervous and Mental Disease, 181, 467-474.

Dube, S., Anda, R., Felitti, V., Chapman, D., Williamson, D., \& Giles, W. (2001). Childhood abuse, household dysfunction, and the risk of attempted suicide throughout the life span: Findings from the adverse childhood experiences study. JAMA, 286, 3089-3096.

Dube, S., Anda, R., Felitti, V., Edward, V., \& Croft, J. (2002). Adverse childhood experiences and personal alcohol abuse as an adult. Addictive Behaviors, 27, 713-725.

Dube, S., Anda, R., Whitfield, C., Brown, D., Felitti, V., Dong, M., et al. (2005). Long-term consequences of childhood sexual abuse by gender of victim. American Journal of Preventive Medicine, 28, 430-438.

Dube, S., Miller, J., Brown, D., Giles, W., Felitti, V., Dong, M., et al. (2006). Adverse childhood experiences and the association with ever using alcohol and initiating alcohol use during adolescence. Journal of Adolescent Health, 28, 444e1-444e10.

Evans, E., Hawton, K., Rodham, K., \& Deeks, J. (2005). The prevalence of suicidal phenomena in adolescents: A systematic review of population-based studies. Suicide and Life-Threatening Behavior, 35, 239-250.

Felitti, V. J., Anda, R. F., Nordenberg, D., Williamson, D. F., Spitz, A. M., Edwards, V., et al. (1998). Relationship of childhood abuse and household dysfunction to many of the leading causes of death in adults the adverse childhood experiences (ACE) study. American Journal of Preventive Medicine, 14, $245-258$.

Groleger, U., Tomori, M., \& Kocmur, M. (2003). Suicidal ideation in adolescence - an indicator of actual risk? Israel Journal of Psychiatry and Related Sciences, 40, 202-208.

Groschwitz, R., Kaess, M., Fischer, G., Ameis, N., Schulze, U., Brunner, R., et al. (2015). The association of non-suicidal self-injury and suicidal behavior according to DSM-5 in adolescent psychiatric inpatients. Psychiatry Research, 228, 454-461.

Hardt, J., Herke, M., \& Schier, K. (2011). Suicidal Ideation, parent-child relationships, and adverse childhood experiences: A cross - validation study using a Graphical Markov Model. Child Psychiatry \& Human Development, 42, 119-133.

Hawton, K., Saunders, K., \& O'Connor, R. (2012). Self - harm and suicide in adolescents. The Lancet, 379, $2373-2382$.

Isohookana, R., Riala, K., Hakko, H., \& Räsänen, P. (2012). Adverse childhood experiences and suicidal behavior of adolescent psychiatric inpatients. European Child \& Adolescent Psychiatry, 22, 13-22.

Johnson, J. H., \& McCutcheon, S. M. (1980). Assessing life stress in older children and adolescents: Preliminary findings with the Life Events Checklist. In J. G. Sarason, \& C. D. Spielberger (Eds.). Stress and anxiety (pp. 111-112). Washington DC: Hemisphere Publishing.

Johnson, J., Cohen, P., Gould, M., Kasen, S., Brown, J., \& Brook, J. (2002). Childhood adversities, interpersonal difficulties, and risk for suicide attempts during late adolescence and early adulthood. Archives of General Psychiatry, 59, 741.

Kaufman, J., Birmaher, B., Brent, D., Rao, U., Flynn, C., Moreci, P., et al. (1997). Schedule for affective disorders and schizophrenia for school-Age children-Present and lifetime version (K-SADS-PL): Initial reliability and validity data. Journal of The American Academy of Child and Adolescent Psychiatry, 36, 980-988.

Kessler, R., Borges, G., \& Walters, E. (1999). Prevalence of and risk factors for lifetime suicide attempts in the National Comorbidity Survey. Archives of General Psychiatry, 56, 617.

Kumar, S., \& George, B. (2013). Life events, social support, coping strategies, and quality of life in attempted suicide. Indian Journal of Psychiatry, 55, 46-51.

Laukkanen, E., Peiponen, S., Halonen, P., Aivio, A., \& Viinamäki, H. (1999). Discriminant validity of the offer self-Image questionnaire in finnish 13-year-old adolescents. Nordic Journal of Psychiatry, 53, 197-201.

Laukkanen, E., Halonen, P., Aivio, A., Viinamäki, H., \& Lehtonen, J. (2000). Construct validity of the Offer Self-Image Questionnaire in Finnish 13-year-old adolescents: Differences in the self-images of boys and girls. Nordic Journal of Psychiatry, 54, 431-435.

Laukkanen, E., Honkalampi, K., Hintikka, J., Hintikka, U., \& Lehtonen, J. (2005). Suicidal ideation among help-seeking adolescents: Association with a negative selfimage. Archives of Suicide Research, 9, 45-55.

Mehlum, L., Ramberg, M., Tormoen, A., Haga, E., Diep, L., Stanley, B., et al. (2016). Dialectical Behavior therapy compared with enhanced usual care for adolescents with repeated suicidal and self-harming behavior: Outcomes over a one-year follow-up. Journal of the American Academy of Child \& Adolescent Psychiatry, 55, 295-300.

Miller, A., Esposito-Smythers, C., Weismoore, J., \& Renshaw, K. (2013). The relation between child maltreatment and adolescent suicidal behavior: A systematic review and critical examination of the literature. Clinical Child and Family Psychology Review, 16, 146-172.

Nock, M., Green, J., Hwang, I., McLaughlin, K., Sampson, N., Zaslavsky, A., et al. (2013). Prevalence, correlates, and treatment of lifetime suicidal behavior among adolescents: Results from the National Comorbidity Survey Replication Adolescent Supplement. JAMA Psychiatry, 9, 1-11.

Offer, D., Ostrov, E., Howard, K., \& Dolan, S. (1992). Offer self-Image questionnaire, revised (OSIQ-R), manual. Los Angeles, California: Western Psychological Services.

Ougrin, D., Tranah, T., Stahl, D., Moran, P., \& Asarnow, J. (2015). Therapeutic interventions for suicide attempts and self-harm in adolescents: Systematic review and meta-analysis. Journal of the American Academy of Child \& Adolescent Psychiatry, 54, 97-107.

Pelkonen, M., Karlsson, L., \& Marttunen, M. (2011). Adolescent suicide: Epidemiology, Psychological theories, risk factors, and prevention. Current Pediatric Reviews, 7 , $52-67$.

Perez, N., Jennings, W., Piquero, A., \& Baglivio, M. (2016). Adverse Childhood Experiences and suicide attempts: The Mediating influence of personality development and problem behaviors. Journal of Youth and Adolescence, 8, 1527-1545.

Preacher, K., \& Hayes, A. (2008). Asymptotic and resampling strategies for assessing and comparing indirect effects in multiple mediator models. Behavior Research Methods, 40, 879-891.

Randall, J., Doku, D., Wilson, M., \& Peltzer, K. (2014). Suicidal behaviour and related risk factors among school-aged youth in the republic of Benin. PUBLIC LIBRARY OF SCIENCE. http://dx.doi.org/10.1371/journal.pone.0088233 [Retrieved from].

Rasmussen, C., Nielsen, L., Petersen, D., Christiansen, E., \& Bielnberg, N. (2013). Adverse life events as risk factors for behavioural and emotional problems in a 7-year follow-up of a population-based child cohort. Nordic Journal of Psychiatry. http://dx.doi.org/10.3109/08039488.2013.794473 Retrieved from.

Reinert, D., \& Allen, J. (2002). The alcohol use identification test (AUDIT): A review of resent research. Alcoholism, Clinical and Experimental Research, 26, 272-279.

Rothman, E., Edwards, E., Heeren, T., \& Hingson, R. (2008). Adverse childhood experiences predict earlier age of drinking onset: results from a representative US sample of current or former drinkers. Pediatrics, 122, e294-e297.

Rytilä-Manninen, M., Lindberg, N., Haravuori, H., Kettunen, K., Marttunen, M., Joukamaa, M., et al. (2014). Adverse childhood experiences as risk factors for serious mental disorders and inpatient hospitalization among adolescents. Child Abuse \& Neglect, 38, 2021-2032.

Rytilä-Manninen, M., Fröjd, S., Haravuori, H., Lindberg, N., Marttunen, M., Kettunen, K., et al. (2016). Psychometric properties of the Symptom Checklist-90 in adolescent psychiatric inpatients and age- and gender - matched community youth. Child and Adolescent Psychiatry and Mental Health, $10,23$.

Saunders, J., Aasland, O., Babor, T., de la Fuente, J., \& Grant, M. (1993). Development of the alcohol use disorders identification test (AUDIT): WHO collaborative project on early detection of persons with harmful alcohol consumption-II. Addiction, 88, 791-804.

Serafini, G., Muzio, C., Piccini, G., Flouri, E., Ferrigno, G., Pompili, M., et al. (2015). Life adversities and suicidal behavior in young individuals: A systematic review. European Child and Adolescent Psychiatry, 24, 1423-1446.

Shonkoff, J. P., \& Garner, A. S. (2012). American academy of pediatrics committee on psychosocial aspects of child and family health; committee on early childhood adoption, and dependent care; section on developmental and behavioral pediatrics. the lifelong effects of early childhood adversity and toxic stress. Pediatrics, 129(1), e232-e246.

Spirito, A., \& Esposito-Smythers, C. (2006). Attempted and completed suicide in adolescence. Annual Review of Clinical Psychology, 2, $237-266$.

Stanley, B., Brown, G., Brent, D., Wells, K., Poling, K., Curry, J., et al. (2009). Cognitive-behavioral therapy for suicide prevention (CBT-SP): Treatment model, feasibility: and acceptability. Journal of the American Academy of Child and Adolescent Psychiatry, 48, 1005-1013. 
Stewart, J., Esposito, E., Glenn, C., Gilman, S., Pridgen, B., Gold, J., et al. (2017). Adolescent self-injurers: Comparison non-ideators, suicide ideators, and suicide attempters. Journal of Psychiatric Research, 84, 105-112.

Thompson, R., Proctor, L., English, D., Dubowitz, H., Narasimhan, S., \& Everson, M. (2012). Suicidal ideation in adolescence: Examining the role of recent adverse experiences. Journal of Adolecence, 35, 175-186.

Tuisku, V., Pelkonen, M., Karlsson, L., Kiviruusu, O., Holi, M., Ruuttu, T., et al. (2006). Suicidal ideation, deliberate self-harm behaviour and suicide attempts among adolescent outpatients with depressive mood disorders and comorbid axis I disorders. European Child \& Adolescent Psychiatry, 15, $199-206$.

Vander Stoep, A., McCauley, E., Flynn, C., \& Stone, A. (2009). Thoughts of death and suicide in early adolescence. Suicide and Life-Threatening Behavior, 39 , 599.

Zouk, H., Tousingnant, M., Sequin, M., Lesage, A., \& Turecki, G. (2006). Characyerization of impulsivity in suicide completers: Clinical behavioral and psychosocial dimensions. Journal of Affective Disorders, 92, 195-204. 